\section{Korrelationskoeffizient, Intraklass-}

R.-D. Hilgers ${ }^{1}$, N. Heussen ${ }^{1}$ und S. Stanzel ${ }^{2}$

${ }^{1}$ Institut für Medizinische Statistik, Universitätsklinikum der RWTH Aachen, Aachen, Deutschland

${ }^{2}$ DKFZ Heidelberg, Heidelberg, Deutschland

Synonym(e) ICC; Intraklass-Korrelation

Englischer Begriff intraclass correlation coefficient (ICC)

Definition Der Intraklass-Korrelationskoeffizient (ICC) beurteilt die Stärke und Richtung der Korrelation (Zusammenhang) zwischen den wiederholten Messungen eines Merkmals am selben Individuum.

Beschreibung Der ICC wird häufig als ein Maß für die Reliabilität einer Messreihe angeben. Zu dessen Berechnung benötigt man zu verschiedenen Zeitpunkten an verschiedenen Individuen erhobene Daten einer Messmethode. Der ICC ist definiert als Quotient der $>$ Varianz zwischen den Individuen und der Summe der Varianzen zwischen und innerhalb der Individuen. Werden an $\mathrm{k}$ Individuen $\mathrm{n}$ zufällige Messungen verglichen, so ist zur Beschreibung der Daten ein einfakto- rielles varianzanalytisches Modell ( $\triangleright$ Varianzanalyse) mit einem Zufallseffekt angemessen. Dann wird der ICC geschätzt durch die Differenz der mittleren Quadratsumme zwischen den Individuen und der mittleren Quadratsumme innerhalb der Individuen dividiert durch die Summe der mittleren Quadratsumme zwischen den Individuen und $(\mathrm{n}-1)$ mal der mittleren Quadratsumme innerhalb der Individuen. Eine analoge Formulierung ergibt sich für zweifaktorielle varianzanalytische Modelle.

Der ICC berechnet sich aus den geschätzten Varianzkomponenten. Im einfaktoriellen varianzanalytischen Modell erlaubt der ICC die Bewertung der absoluten Übereinstimmung der wiederholten Messungen. Im zweifaktoriellen Modell erlaubt der ICC die Bewertung der Konsistenz bzw. der absoluten Übereinstimmung - nach Einführung eines Korrekturterms im Nenner des Quotienten - zwischen den Messmethoden ( $\triangleright$ Übereinstimmung zweier Messmethoden).

\section{Literatur}

Hartung J, Elpelt B, Klösener KH (1995) Statistik - Lehr- und Handbuch der angewandten Statistik. Oldenbourg Verlag, München

Shoukri MM (2004) Measures of interobserver agreement. Chapman \& Hall, Boca Raton 